\title{
CAPACITATED WAREHOUSE LOCATION PROBLEMS WITH ECONOMIES OF SCALE IN OPERATING COSTS
}

\author{
Birgit Schildt, TH Darmstadt
}

In most industries distribution costs represent a major expense, so that a careful design of a company's distribution system appears to be of vital importance. Relevant costs to be considered concern the location of the distributing facilities as well as the allocation of customers to those locations chosen. Minimization of distribution costs focuses on a tradeoff between transportation costs favouring decentralisation and operating costs associated with the facilities, where a potential loss of economies favours centralization. Realistic assumptions about the underlying cost structure are an important precondition for obtaining a reasonable solution.

Though economies of scale are very common in real life production-transportation planning situations, most publications concerning nonlinear discrete location problems consider some type of convex objective function. This may be encouraged by the fact, that in convex minimization any local optimal solution is known to be a global optimum, which makes things a lot easier. Nevertheless, in most practical applications it is more realistic to assume decreasing marginal operating costs associated with each potential site. Respective models lead to concave minimization problems, that so far have only been discussed by a lew authors.

Considering the problem of finding a minimal total costs warehouse design wherein individual warehouses have limited capacity and exhibit continuous economies of scale in variable warehousing costs, we investigate a number of different exact and heuristic solution procedures. Heuristic methods include a cross decomposition approach for deriving good upper and lower bounds on the optimal objective function value as well as the application of recent improvement metastrategies. Besides, a branch and bound procedure is introduced, where lower bounds are obtained via Lagrangean relaxation; the relaxed subproblems may be solved to optimality by inspection.

Presentation includes a comparison of respective numerical results. Test data were generated reflecting different real world cost situations. 